\title{
CDK5RAP1 deficiency induces cell cycle arrest and apoptosis in human breast cancer cell line by the ROS/JNK signaling pathway
}

\author{
HONGBIN WANG, LI WEI, CHANGLI LI, JING ZHOU and ZHIGAO LI
}

\author{
Department of Breast Surgery, Harbin Medical University Cancer Hospital, Harbin, Heilongjiang 150081, P.R. China
}

Received July 8, 2014; Accepted August 21, 2014

DOI: $10.3892 /$ or.2015.3736

\begin{abstract}
Cyclin-dependent kinase 5 regulatory subunit associated protein 1 (CDK5RAP1) is an enzyme which post-synthetically converts the RNA modification N6-isopentenyladenosine $\left(\mathrm{i}^{6} \mathrm{~A}\right)$ into 2-methylthio-N6-isopentenyladenosine $\left(\mathrm{ms}^{2} \mathrm{i}^{6} \mathrm{~A}\right)$. However, the interaction between CDK5RAP1 deficiency and cell apoptosis has not been studied. Breast cancer has long been a leading cause of mortality in the world. Therefore, in the present study, CDK5RAP1 deficiency in a human breast cancer cell line was investigated. CDK5RAP1 small interfering RNA (siRNA) and negative control siRNA were transfected into MCF-7 cells, and the cells were further incubated for $48 \mathrm{~h}$. CDK5RAP1 deficiency suppressed tumor growth in MCF-7 cells and arrested the cells at G2/M phase. CDK5RAP1 deficiency also induced cell apoptosis and reactive oxygen species (ROS) generation. Furthermore, western blot analysis showed that the expression of phospho-c-Jun N-terminal kinase (p-JNK), p53, caspase-9 and caspase-3 were upregulated in CDK5RAP1-deficient MCF-7 cells. Pretreatment with N-acetyl-cysteine (NAC), the inhibitor of ROS, or with SP600125, the inhibitor of JNK, prevented the apoptosis and the high expression of p-JNK, p53, caspase-9 and caspase-3 in CDK5RAP1-deficient MCF-7 cells. Taken together, these data indicated that CDK5RAP1 deficiency induced cell cycle arrest and apoptosis in human breast cancer MCF-7 cells by the ROS/JNK signaling pathway. Our findings indicated a novel therapeutic strategy for cancer.
\end{abstract}

\section{Introduction}

Cyclin-dependent kinase 5 regulatory subunit associated protein 1 (CDK5RAP1) is a radical S-adenosyl methionine (SAM) enzyme (1) with homology to the bacterial MiaB protein (2), which post-synthetically converts the RNA modification

Correspondence to: Dr Zhigao Li, Department of Breast Surgery, Harbin Medical University Cancer Hospital, 6 Baojian Road, Harbin, Heilongjiang 150081, P.R. China

E-mail: lizhigao2014@sina.com

Key words: CDK5RAP1, MCF-7 cell, cell cycle, reactive oxygen species, c-Jun $\mathrm{N}$-terminal kinase
N6-isopentenyladenosine ( $\left.\mathrm{i}^{6} \mathrm{~A}\right)$ into 2-methylthio-N6-isopentenyladenosine ( $\left.\mathrm{ms}^{2} \mathrm{i}^{6} \mathrm{~A}\right)$ at A37 (3), as shown in Fig. 1A. It was discovered to inhibit the active CDK5 kinase and function in codon suppression (4) and stabilization of the codon/anticodon interaction (5). CDK5 aberrant regulation can lead to a number of diseases (6). The biochemical link established by CDK5RAP1 between the enzymatic modification of transfer RNA (tRNA) tanticodon loops and CDK5 kinase activity is highly unusual, particularly since the modified base $\mathrm{ms}^{2} \mathrm{i}^{6} \mathrm{~A}$ is known to exist in tRNA of prokaryotic origin (7), particularly in mitochondrial tRNA of mammals (8).

Breast cancer has long been a leading cause of mortality in women worldwide (9). Due to the limited efficacy of traditional therapy, it is necessary to exploit a new treatment strategy for breast cancer. Mitochondria-initiated responses are thought to be the major pathway for apoptosis, and, therefore, targeting the mitochondria is a novel strategy for cancer therapy (10). Hence, in the present study, we sought to determine if the mistranslation of $\mathrm{ms}^{2} \mathrm{i}^{6} \mathrm{~A}$ in mitochondrial tRNA caused by CDK5RAP1 deficiency affects the human breast cancer cell line, MCF-7 cells.

Cell cycle arrest is an important cause of growth inhibition. Many anticancer agents reduce malignant growth by arresting the cell cycle at the G1, S or G2/M phases (11). Arresting the cell cycle is an effective method to regulate cell cycle progression, and to contribute to malignant cell proliferation (12). Apart from cell cycle arrest, apoptosis is another cause of growth inhibition. There is compelling evidence that excessive reactive oxygen species (ROS) production surmounts cellular antioxidant defenses, triggering apoptosis (13), and cancer cells are more sensitive to rapid increases in ROS levels than normal cells. Oncogenic transformation elevates basal ROS levels significantly so that any further acute increases can trigger reactivation of the apoptotic program in cancer cells (14). Various apoptotic stimuli can rapidly activate MAPKs, which include phospho-c-Jun N-terminal kinase (p-JNK) (15). The activation of JNK is associated with ROS elevation (16). The p-JNK activated through ROS-dependent pathway induces the overexpression of tumor suppressors, such as p53, then leads to cell apoptosis (17).

In the present study, to the best of our knowledge, the hypothesis that CDK5RAP1 deficiency inhibits tumor growth in a human breast cancer cell line was explored for the first time. The results showed that CDK5RAP1 deficiency induced MCF-7 cell cycle arrest and apoptosis, which could be 
A

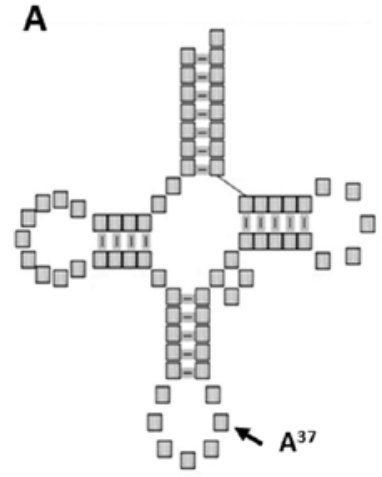<smiles>[R]n1cnc2c(NCC=C(C)C)ncnc21</smiles>

$\mathrm{i}^{6} \mathrm{~A}$<smiles>CSc1nc(NCC=C(C)C)c2ncn(P)c2n1</smiles>

$m s^{2} i^{6} \mathrm{~A}$

B

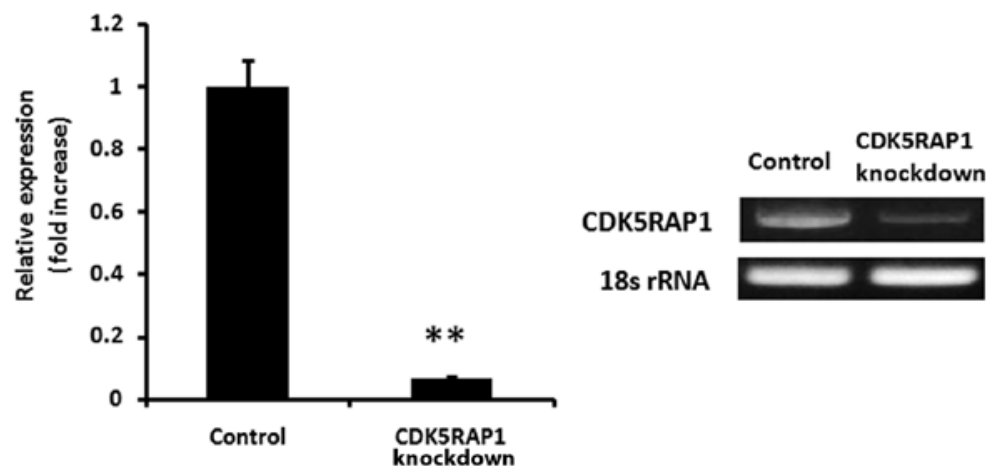

Figure 1. (A) The structure of transfer RNA (tRNA) and the effect of cyclin-dependent kinase 5 regulatory subunit associated protein 1 (CDK5RAP1). tRNA is the most intensively modified small RNA, and CDK5RAP1 is an enzyme which post-synthetically converts the tRNA modification N6-isopentenyladenosine $\left(i^{6} \mathrm{~A}\right)$ into 2-methylthio-N6-isopentenyladenosine $\left(\mathrm{ms}^{2} \mathrm{i}^{6} \mathrm{~A}\right)$ at A37. (B) Confirmation of CDK5RAP1 deficiency. MCF-7 cells were seeded onto 6-well plates at the density of $1 \times 10^{5}$ cells/well and grown to 60-80\% confluence. CDK5RAP1 small interfering RNA (siRNA) and non-targeted negative control siRNA were transfected into MCF-7 cells. Quantitative polymerase chain reaction (qPCR) was used to confirm the result of siRNA transfection. CDK5RAP1 was significantly knocked down by siRNA transfection compared with the control group. Data are expressed as the mean \pm standard deviation $(\mathrm{n}=5)$. $\mathrm{P}<0.05$ was considered to indicate a statistically significant difference $\left({ }^{* *} \mathrm{P}<0.01\right)$.

prevented by the pretreatment with $\mathrm{N}$-acetyl-cysteine (NAC; the inhibitor of ROS), or SP600125 (the inhibitor of JNK), suggesting that the ROS/JNK signaling pathway is an important mechanism in the apoptosis process. Our study indicated that this may be a novel therapeutic strategy for cancer.

\section{Materials and methods}

Cell culture. The human breast cancer cell line MCF-7 was purchased from the American Type Culture Collection (ATCC; Manassas, VA, USA). This study was performed in accordance with the Experiment Guidelines of Harbin Medical University (Harbin, China) and ethical approval was obtained from Harbin Medical University. MCF-7 cells were cultured in RPMI-1640 medium supplemented with $10 \%$ fetal bovine serum (FBS), $100 \mathrm{U} / \mathrm{ml}$ penicillin and $100 \mathrm{mg} / \mathrm{ml}$ streptomycin (GIBCO, Grand Island, NY, USA), and were cultured in an incubator (Sanyo, Tokyo, Japan) with $5 \% \mathrm{CO}_{2}$ at $37^{\circ} \mathrm{C}$.

Small interfering RNA (siRNA) transfection. CDK5RAP1 siRNA and non-targeted negative control siRNA were purchased from Santa Cruz Biotechnology Inc. (Santa Cruz, CA, USA). MCF-7 cells were seeded onto 6-well plates at the recommended density ( $1 \times 10^{5}$ cells/well) and grown to $60-80 \%$ confluence prior to transfection. siRNAs were transfected into
MCF-7 cells with siRNA Transfection Reagent (Santa Cruz Biotechnology Inc.) according to the manufacturer's instructions. MCF-7 cells were further incubated for another $48 \mathrm{~h}$ and then used for experiments.

Quantitative polymerase chain reaction ( $q P C R$ ). CDK5RAP1 siRNA and negative control siRNA were transfected into MCF-7 cells, and the cells were further incubated for $48 \mathrm{~h}$. Total RNA was extracted from MCF-7 cells and relative mRNA was normalized to 18s. The following primers (Hokkaido System Science Co. Ltd. Sapporo, Japan) were used: CDK5RAP1 forward, 5'-ATGGCTGCCAGATGAATG TGA-3' and reverse, 5'-CTCTTGGAGGTTACTGGTCCG-3'; 18s forward, 5'-GTAACCCGTTGAACCCCATT-3' and reverse, 5'-CCATCCAATCGGTAGTAGCG-3'. qPCR was performed using the ABI 7300 Fast real-time PCR system (Applied Biosystems, Foster City, CA, USA).

3-(4,5-Dimethylthiazol-2-yl)-2,5-diphenyltetrazolium bromide (MTT) assay. The viability of normal MCF-7 cells and CDK5RAP1-deficient MCF-7 cells was determined by a colorimetric MTT assay according to the method described previously (18). Absorbance at $550 \mathrm{~nm}$ was determined by an MTP-800 microplate reader (Corona Electric, Tokyo, Japan). Absorbance at $690 \mathrm{~nm}$ was also measured to compensate for 
any interfering effects of cell debris and the microtiter plate. Percentage of viable cell number was calculated as: Optical density (OD) of treated sample/OD of untreated control x100.

Cell cycle analysis. CDK5RAP1 siRNA and negative control siRNA were transfected into MCF-7 cells, and the cells were further incubated for $48 \mathrm{~h}$. Then, MCF-7 cells were trypsinized and fixed in $99 \%$ ethanol at $-20^{\circ} \mathrm{C}$ for $2 \mathrm{~h}$, washed and re-suspended in $420 \mu \mathrm{l}$ PBS. Subsequently, samples were first incubated with RNase A (Sigma, Shanghai, China) (50 $\mu \mathrm{l}$ of a $10 \mathrm{mg} / \mathrm{ml}$ solution) at $37^{\circ} \mathrm{C}$ for $30 \mathrm{~min}$, and then PI (20 $\mu \mathrm{l}$ of a $0.2 \mathrm{mg} / \mathrm{ml}$ solution) at room temperature for $10 \mathrm{~min}$. DNA content was analyzed by flow cytometry using a FACSCalibur and CellQuest software (Becton Dickinson, Franklin Lake, NJ, USA), as previously described (19).

Apoptosis assay. MCF-7 cell apoptosis staining was performed using an Annexin V (cell apoptosis signaling component)Biotin Apoptosis kit as per the manufacturer's instructions (Mountain View, CA, USA). The MCF-7 cells were seeded in a 6 -well plate at the density of $1 \times 10^{5}$ cells/well and were pretreated or non-treated with NAC $(5 \mathrm{mM})$ or SP600125 $(5 \mu \mathrm{M})$ (Sigma) for $1 \mathrm{~h}$ prior to CDK5RAP1 siRNA transfection. After transfection with CDK5RAP1 siRNA or control siRNA, MCF-7 cells were further incubated for $48 \mathrm{~h}$. Stained cells were analyzed using FACSCalibur ${ }^{\mathrm{TM}}$ Flow Cytometry (BD Biosciences, San Jose, CA, USA) with CellQuest software. Ten thousand events were collected for each sample.

Nuclear staining with Hoechst 33342 for morphological evaluation. MCF-7 cells were plated in 6-well plates at the density of $1 \times 10^{5}$ cells/well. CDK5RAP1 siRNA and negative control siRNA were transfected into MCF-7 cells, and the cells were further incubated for $48 \mathrm{~h}$. Then, the cells were washed with PBS, fixed in $4 \%$ paraformaldehyde (Bioss, Beijing, China) for $30 \mathrm{~min}$ and then stained with $20 \mathrm{mg} / \mathrm{ml}$ Hoechst 33342 for $15 \mathrm{~min}$ at room temperature in the dark. Cells were then assessed by fluorescence microscopy for morphological changes.

Detection of intracellular ROS. Intracellular accumulation of ROS was estimated using the fluorescent dye $\mathrm{H}_{2}$-DCFDA (Life Technologies, Tokyo, Japan), which is converted to a membrane impermeable and highly fluorescent compound, dichlorofluorescin diacetate (DCF), in the cell in the presence of ROS (20). The MCF-7 cells were seeded in a 6-well plate at the density of $1 \times 10^{5}$ cells/well. Following transfection with CDK5RAP1 siRNA or control siRNA, MCF-7 cells were further incubated for $48 \mathrm{~h}$. The cells were rinsed with a serum-free medium and were incubated in $5 \mu \mathrm{M} \mathrm{H}_{2}$-DCFDA for $60 \mathrm{~min}$ at $37^{\circ} \mathrm{C}$. The cells were then examined under a fluorescence microscope (C1-T-SM; Nikon, Tokyo, Japan), collected and subjected to a fluorescence spectrophotometer (F-2500; Hitachi, Tokyo, Japan) to detect the fluorescence of DCF inside cells (excitation, $488 \mathrm{~nm}$; emission, $521 \mathrm{~nm}$ ).

Flow cytometry. Intracellular ROS was measured using 2',7'-dichlorodihydrofluorescein diacetate (DCFH-DA) (Life Technologies). The MCF-7 cells were seeded in a 6-well plate at the density of $1 \times 10^{5}$ cells/well. CDK5RAP1 siRNA and negative control siRNA were transfected into MCF-7 cells, and the cells were further incubated for $48 \mathrm{~h}$. MCF-7 cells were then washed with PBS and labeled with $10 \mu \mathrm{M}$ DCFDA for $30 \mathrm{~min}$. Then, excess DCFH-DA was removed by washing the cells in serum-free RPMI-1640 medium (Sigma). The fluorescence intensities were measured using a FACSCalibur flow cytometer (BD Biosciences).

Western blot analysis. Electrophoresis was performed using a vertical slab gel with $12 \%$ polyacrylamide content according to the method described previously (21). The transfer of proteins from the SDS polyacrylamide gel to a membrane was performed electrophoretically according to the method described previously (22) with certain modifications using a Semi Dry Electroblotter (Sartorius AG, Goettingen, Germany) for 90 min with an electric current of $15 \mathrm{~V}$. The membrane was treated with Block Ace ${ }^{\mathrm{TM}}(4 \%)$ for $30 \mathrm{~min}$ at $22^{\circ} \mathrm{C}$. The first reaction was performed using rabbit immunoglobulin (IG) G antibodies against JNK, p-JNK, p53, caspase-9 and caspase-3 (Sigma) in PBS containing $0.03 \%$ Tween-20 for $1 \mathrm{~h}$ at $22^{\circ} \mathrm{C}$. Following washing in the same buffer, the second reaction was performed using horseradish peroxidase (HRP)conjugated anti-rabbit goat $\operatorname{IgG}(20 \mathrm{ng} / \mathrm{ml})$ for $30 \mathrm{~min}$ at $22^{\circ} \mathrm{C}$. After washing, the enhanced chemiluminescence (ECL) reaction was performed on the membrane using the ECL Plus Western Blotting Detection System ${ }^{\mathrm{TM}}$ (GE Healthcare Life Sciences).

Statistical analysis. Data are expressed as the mean \pm standard deviation. Each experiment was repeated at least 3 times. The Student's t-test was used and $\mathrm{P}<0.05$ was considered to indicate a statistically significant difference.

\section{Results}

CDK5RAP1 deficiency suppresses tumor growth in MCF-7 cells. To investigate the effect of CDK5RAP1 deficiency on the growth of human breast cancer cell line (MCF-7 cells), MCF-7 cells were seeded onto 6 -well plates at the recommended density $\left(1 \times 10^{5}\right.$ cells/well) and grown to $60-80 \%$ confluence prior to transfection. CDK5RAP1 siRNA and negative control siRNA were transfected into MCF-7 cells, and then the cells were further incubated for $48 \mathrm{~h}$. The viability of normal MCF-7 cells and CDK5RAP1-deficient MCF-7 cells was determined by a colorimetric MTT assay. The tumor growth was significantly suppressed in the CDK5RAP1-deficient MCF-7 cells (Fig. $2 \mathrm{~A}$ and $\mathrm{B} ; \mathrm{P}<0.01$ ).

CDK5RAP1 deficiency arrests MCF-7 cells at the G2/M phase. CDK5RAP1 siRNA and negative control siRNA were transfected into MCF-7 cells, and then the cells were further incubated for $48 \mathrm{~h}$. Cell cycle analysis was performed using a FACSCalibur and CellQuest software. CDK5RAP1 deficiency arrested MCF-7 cells at the G2/M phase significantly compared with control MCF-7 cells (Fig. 2C and D; $\mathrm{P}<0.01$ ).

CDK5RAP1 deficiency induces MCF-7 cell apoptosis. MCF-7 cells were plated in 6-well plates at the density of 
A
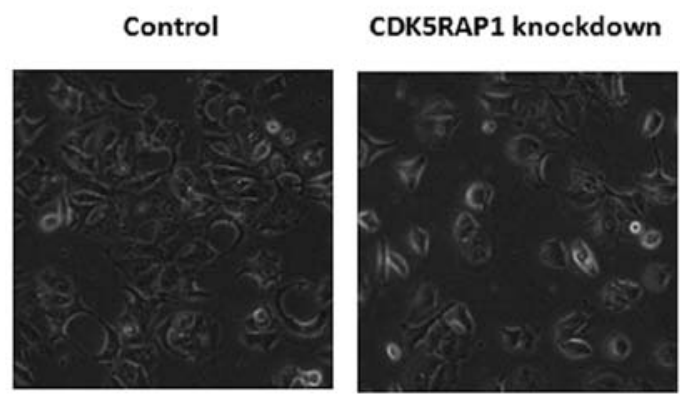

C

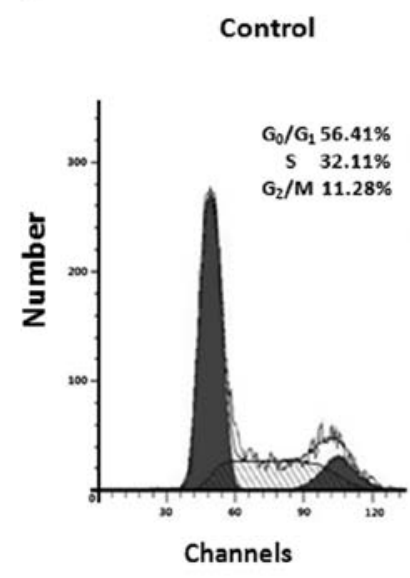

B

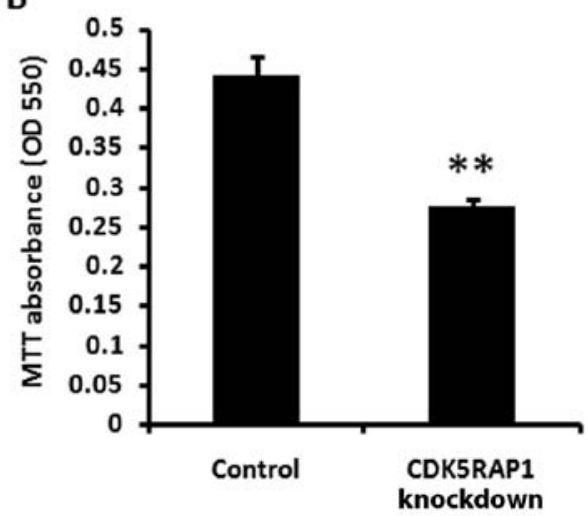

D

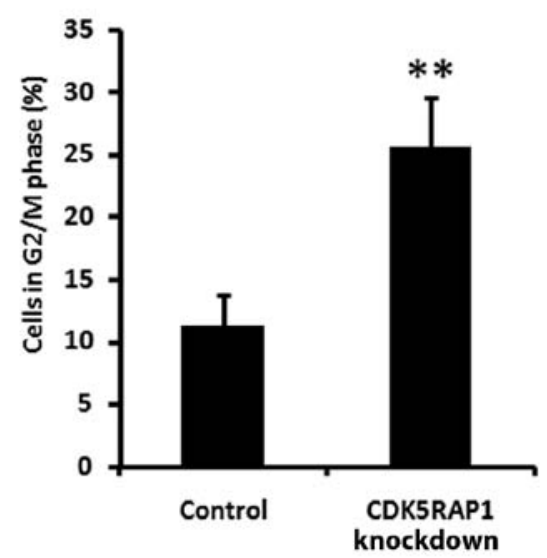

Figure 2. Cyclin-dependent kinase 5 regulatory subunit associated protein 1 (CDK5RAP1) deficiency suppresses tumor growth in MCF-7 cells and arrests the cells at G2/M phase. MCF-7 cells were seeded in a 6-well plate at the density of 1x105 cells/well. CDK5RAP1 small interfering RNA (siRNA) and negative control siRNA were transfected into MCF-7 cells, and the cells were further incubated for $48 \mathrm{~h}$. (A) Image of MCF-7 cells. MCF-7 cells were counted and photographed under a phase-contrast microscope at high-power field (x400 magnification). (B) Viability of MCF-7 cells was determined by MTT assay. CDK5RAP1 deficiency significantly suppressed tumor growth in MCF-7 cells. (C) Cell cycle analysis was used to determine the cell cycle of MCF-7 cells. CDK5RAP1 deficiency arrested the MCF-7 cells at G2/M phase significantly. (D) Quantification of (C). Data are expressed as the mean \pm standard deviation $(\mathrm{n}=5) . \mathrm{P}<0.05$ was considered to indicate a statistically significant difference $\left({ }^{* *} \mathrm{P}<0.01\right)$.

$1 \times 10^{5}$ cells/well. Following transfection with CDK5RAP1 siRNA or control siRNA, MCF-7 cells were further incubated for $48 \mathrm{~h}$. The MCF-7 cell apoptosis was performed using an Annexin V-Biotin Apoptosis kit and nuclear staining with Hoechst 33342 by fluorescence microscopy. CDK5RAP1 deficiency induced MCF-7 cell apoptosis significantly compared with control MCF-7 cells (Fig. 3A and B, Annexin V-Biotin; Fig. $3 \mathrm{C}$ and $\mathrm{D}$, Hoechst 33342 staining. $\mathrm{P}<0.01)$.

CDK5RAP1 deficiency induces ROS generation in MCF-7 cells. CDK5RAP1 siRNA and negative control siRNA were transfected into MCF-7 cells, and then the cells were further incubated for $48 \mathrm{~h}$. Intracellular accumulation of ROS was estimated using the fluorescent dye $\mathrm{H}_{2}$-DCFDA (Fig. 3E and G), and flow cytometry using DCFH-DA (Fig. 3F). CDK5RAP1 deficiency significantly induced ROS generation in MCF-7 cells $(\mathrm{P}<0.01)$.

CDK5RAPI deficiency upregulates the expression of p-JNK, p53, caspase-9 and caspase-3 in MCF-7 cells. MCF-7 cells were plated in 6 -well plates at the density of $1 \times 10^{5}$ cells/well. After transfection with CDK5RAP1 siRNA or control siRNA, MCF-7 cells were further incubated for $48 \mathrm{~h}$. The expression levels of p-JNK, p53, caspase-9 and caspase-3 in MCF-7 cells were measured by western blot analysis. CDK5RAP1 deficiency upregulated the expression of p-JNK, p53, caspase-9 and caspase-3 significantly compared with control MCF-7 cells. $\beta$-actin was used as the normalization (Fig. 4).

NAC and SP600125 prevent MCF-7 cell apoptosis induced by CDK5RAP1 deficiency. The MCF-7 cells were seeded in a 6 -well plate at the density of $1 \times 10^{5}$ cells/well and were pretreated or non-treated with NAC $(5 \mathrm{mM})$ or SP600125 $(5 \mu \mathrm{M})$ for $1 \mathrm{~h}$ prior to CDK5RAP1 siRNA transfection. Following transfection with CDK5RAP1 siRNA or control siRNA, MCF-7 cells were further incubated for $48 \mathrm{~h}$. CDK5RAP1 deficiency induced MCF-7 cell apoptosis significantly compared with control MCF-7 cells, while pretreatment with NAC or SP600125 prevented the CDK5RAP1 deficiencyinduced apoptosis significantly (Fig. 5A and B; P<0.01). 
A

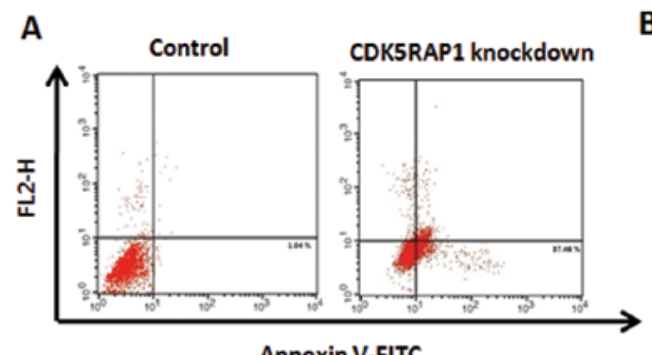

Annexin V-FITC

C

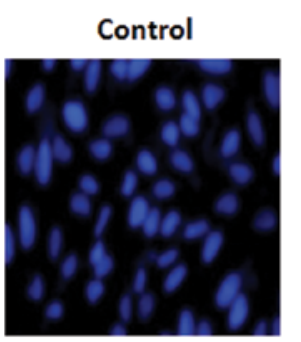

E

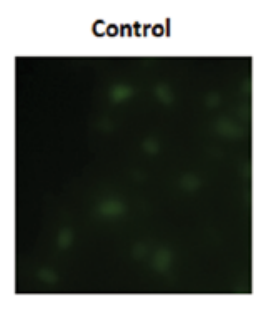

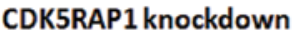

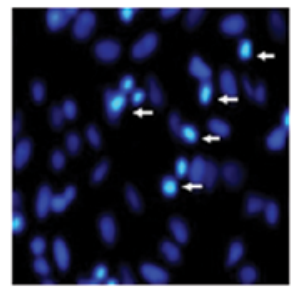

F

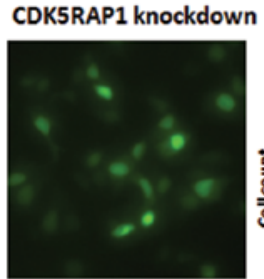

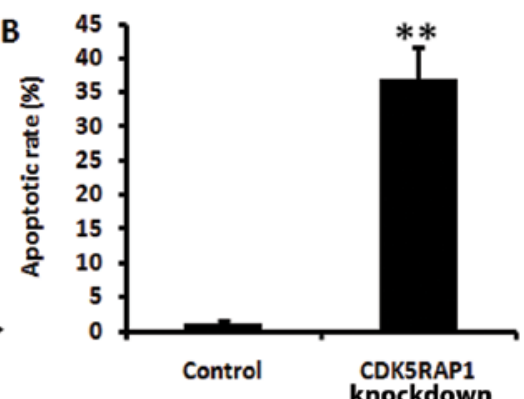

D

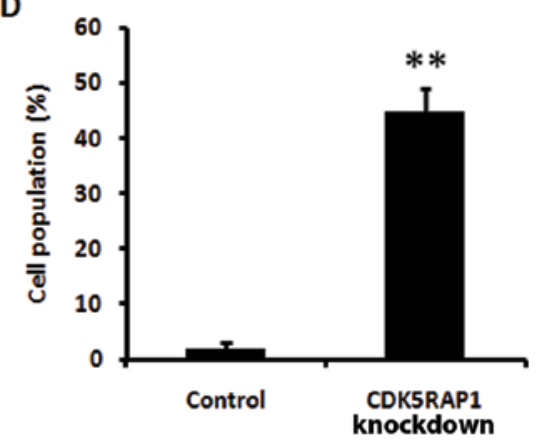

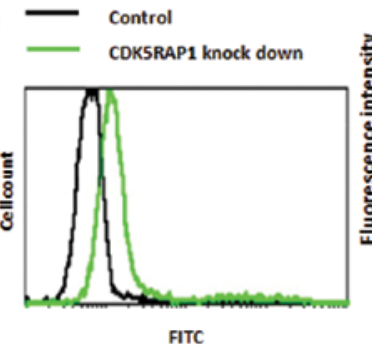

G

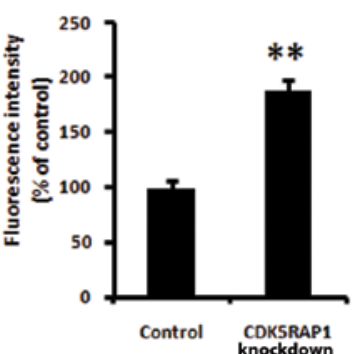

Figure 3. Cyclin-dependent kinase 5 regulatory subunit associated protein 1 (CDK5RAP1) deficiency induces cell apoptosis and reactive oxygen species (ROS) generation in MCF-7 cells. MCF-7 cells were seeded in a 6-well plate at the density of 1x105 cells/well. CDK5RAP1 small interfering RNA (siRNA) and negative control siRNA were transfected into MCF-7 cells, and the cells were further incubated for $48 \mathrm{~h}$. (A) Annexin V-Biotin Apoptosis kit was used to detect MCF-7 cell apoptosis. (B) Quantification of (A). (C) Nuclear staining with Hoechst 33342 by fluorescence microscopy was used to assess the MCF-7 cell apoptosis. Light blue fluorescence indicates live cells, while dark blue fluorescence indicates apoptotic cells. CDK5RAP1 deficiency induced MCF-7 cell apoptosis significantly compared with control MCF-7 cells. (D) Quantification of (C). (E and F) Intracellular accumulation of ROS was estimated by flow cytometry. CDK5RAP1 deficiency significantly induced ROS generation in MCF-7 cells. (G) Quantification of (E). Data are expressed as the mean \pm standard deviation $(\mathrm{n}=5)$. $\mathrm{P}<0.05$ was considered to indicate a statistically significant difference $\left({ }^{* *} \mathrm{P}<0.01\right)$.

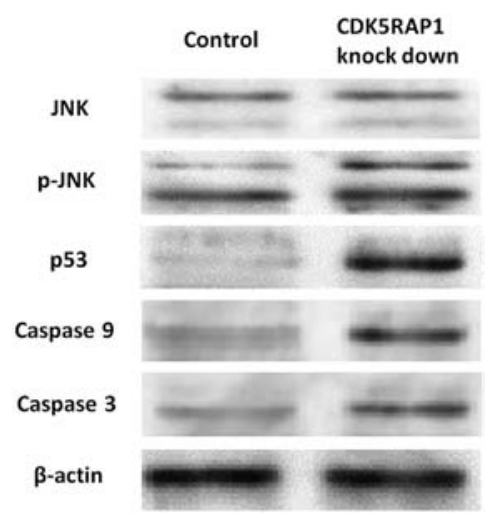

Figure 4. Cyclin-dependent kinase 5 regulatory subunit associated protein 1 (CDK5RAP1) deficiency upregulates the expression of phospho-c-Jun N-terminal kinase (p-JNK), p53, caspase-9 and caspase-3 in MCF-7 cells MCF-7 cells were seeded in a 6-well plate at the density of $1 \times 10^{5}$ cells/well. CDK5RAP1 small interfering RNA (siRNA) and negative control siRNA were transfected into MCF-7 cells, and the cells were further incubated for 48 h. CDK5RAP1 deficiency upregulated the expression of p-JNK, p53, caspase-9 and caspase-3 significantly compared with control MCF-7 cells. $\beta$-actin was used as the normalization.
NAC and SP600125 prevent the CDK5RAPl deficiencyinduced high expression of $p-J N K, p 53$, caspase- 9 and caspase-3 in MCF-7 cells. The MCF-7 cells were pretreated or non-treated with NAC (5 mM) or SP600125 $(5 \mu \mathrm{M})$ for $1 \mathrm{~h}$ prior to CDK5RAP1 siRNA transfection. After transfection with CDK5RAP1 siRNA or control siRNA, MCF-7 cells were further incubated for $48 \mathrm{~h}$. CDK5RAP1 deficiency upregulated the expression of p-JNK, p53, caspase-9 and caspase-3 significantly, while pretreatment with NAC or SP600125 prevented the CDK5RAP1 deficiency-induced high expression of p-JNK, p53, caspase- 9 and caspase-3 in MCF-7 cells. $\beta$-actin was used as the normalization (Fig. 5C).

\section{Discussion}

The present study demonstrated, to the best of our knowledge for the first time, that CDK5RAP1 deficiency suppresses tumor growth and induces cell cycle arrest and apoptosis in a human breast cancer cell line (MCF-7 cells). CDK5RAP1 is a radical SAM enzyme (1) with homology to the bacterial 


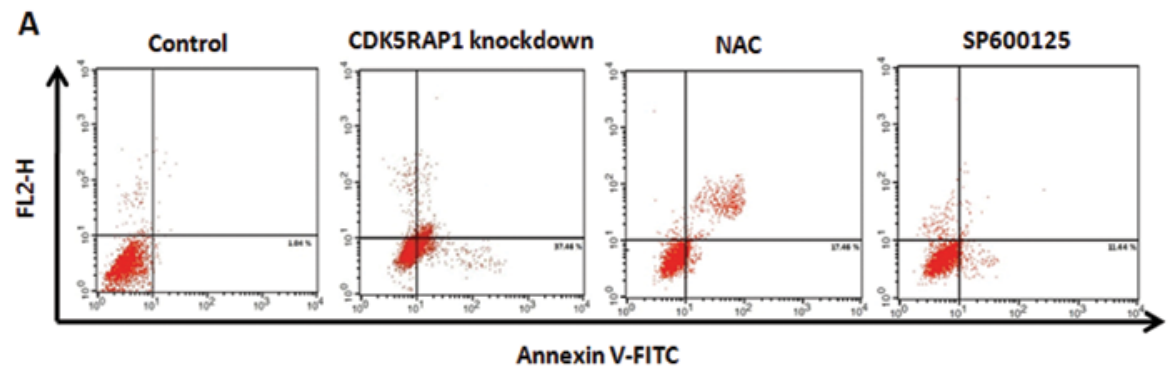

B

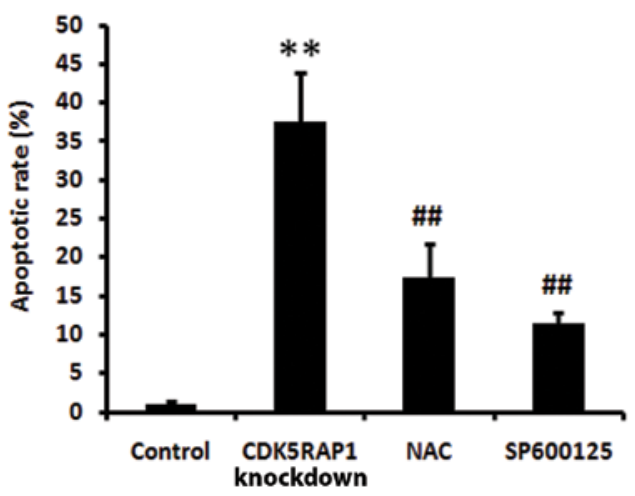

C

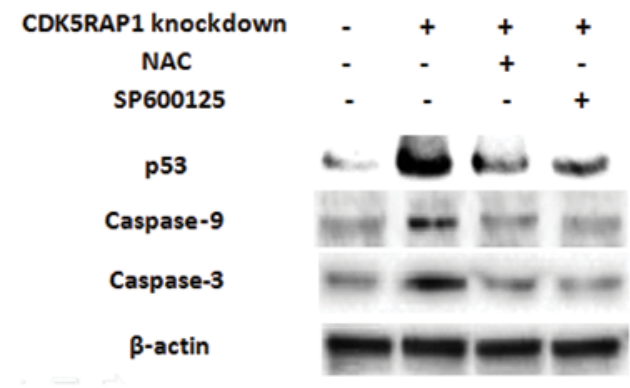

Figure 5. N-acetyl-cysteine (NAC; the inhibitor of ROS) and SP600125 (the inhibitor of JNK) prevent the effect of cyclin-dependent kinase 5 regulatory subunit associated protein 1 (CDK5RAP1) deficiency on MCF-7 cells. The MCF-7 cells were seeded in a 6 -well plate at the density of 1x10 cells/well and were pretreated or non-treated with NAC $(5 \mathrm{mM})$ or SP600125 $(5 \mu \mathrm{M})$ for $1 \mathrm{~h}$ prior to CDK5RAP1 small interfering RNA (siRNA) transfection. After transfection with CDK5RAP1 siRNA or control siRNA, MCF-7 cells were further incubated for $48 \mathrm{~h}$. (A) Pretreatment with NAC or SP600125 prevented the CDK5RAP1 deficiency-induced apoptosis. (B) Quantification of (A). (C) CDK5RAP1 deficiency-induced high expression of phospho-c-Jun N-terminal kinase (p-JNK), p53, caspase-9 and caspase-3 in MCF-7 cells was prevented by the pretreatment with NAC or SP600125. $\beta$-actin was used as the normalization. Data are expressed as the mean \pm standard deviation $(\mathrm{n}=5)$. $\mathrm{P}<0.05$ was considered to indicate a statistically significant difference ${ }^{* *} \mathrm{P}<0.01, \mathrm{CDK} 5 \mathrm{RAP} 1 \mathrm{knockdown}$ vs control; ${ }^{\# \#} \mathrm{P}<0.01$, NAC and SP600125 vs CDK5RAP1 knockdown).

MiaB protein (2), which post-synthetically converts the RNA modification $\mathrm{i}^{6} \mathrm{~A}$ into $\mathrm{ms}^{2} \mathrm{i}^{6} \mathrm{~A}$ (3-6) (Fig. 1A). The biochemical link established by CDK5RAP1 between the enzymatic modification of tRNA tanticodon loops and CDK5 kinase activity is highly unusual, particularly since the modified base $\mathrm{ms}^{2} \mathrm{i}^{6} \mathrm{~A}$ is known to exist in tRNA of prokaryotic origin (7), particularly in mitochondrial tRNA of mammals (8).

Breast cancer has long been a leading cause of mortality in women of developed and developing countries $(9,23)$. Cell cycle arrest is an important cause of growth inhibition. Many anticancer agents exhibit anti-proliferation by inhibiting cell cycle progression at a particular check point such as $\mathrm{G0} / \mathrm{G} 1$, $\mathrm{S}$, or G2/M (11). Deregulation of cell cycle has been linked with cancer initiation and progression (24). Arresting the cell cycle is an effective method to regulate cell cycle progression, and contribute to malignant cell proliferation (12). It has been reported that the expression of CDK5RAP1 gene is related to the regulation and progression of the $\mathrm{M}$ phase of the cell cycle (25). In accordance with that, our present study confirmed that CDK5RAP1 deficiency suppressed tumor growth in MCF-7 cells and arrested the cells at G2/M phase (Fig. 2). Apart from cell cycle arrest, apoptosis is another cause of growth inhibition (26). Apoptosis, or programmed cell death, is an essential mechanism through which many types of chemotherapeutic agents inhibit tumor growth (27). Mitochondria-initiated responses are thought to be the major pathway for apoptosis, and, therefore, targeting the mitochondria is a novel strategy for cancer therapy (10). Our present study also confirmed that CDK5RAP1 deficiency induced MCF-7 cell apoptosis (Fig. 3A-D).

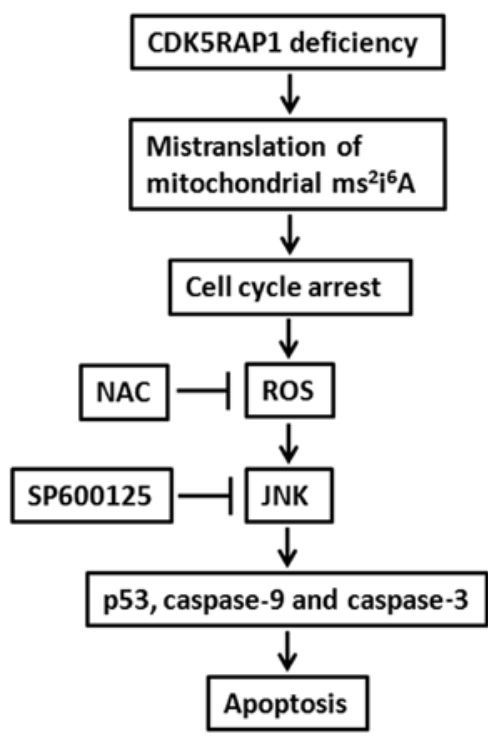

Figure 6. Mechanism of the apoptosis process. Cyclin-dependent kinase 5 regulatory subunit associated protein 1 (CDK5RAP1) deficiency induced cell cycle arrest and apoptosis in MCF-7 cells via reactive oxygen species (ROS) generation, resulting in phospho-c-Jun N-terminal kinase (p-JNK) and p53 activation, increase in the cleavage of caspase- 9 and caspase-3. CDK5RAP1 deficiency induced cell cycle arrest and apoptosis in human breast cancer MCF-7 cells by the ROS/JNK signaling pathway.

ROS, which is the byproduct of normal cellular oxidative processes, has been suggested to regulate the process involved in the initiation of apoptotic signaling (28) and has been implicated in several oncogenic pathways. Although it 
has been reported to be a tumor growth promoter (29), there is compelling evidence that ROS production surmounts cellular antioxidant defenses, triggering apoptosis (13), and cancer cells are more sensitive to rapid increases in ROS levels than normal cells. ROS-mediated cytotoxicity has also been identified as an important mechanism in some anticancer agents (30). Accumulating evidence indicates that many anticancer agents destroy tumor cells by raising the level of ROS above a toxic threshold (31). Oncogenic transformation elevates basal ROS levels significantly so that any further acute increases can trigger reactivation of the apoptotic program in cancer cells (14). High level of ROS can destroy the integrity of plasma membrane, affect dynamic of actin cytoskeleton and cause DNA damage, cumulatively known as oxidative stress (32). To investigate whether CDK5RAP1 deficiency-induced MCF-7 cell apoptosis is promoted through an increase in ROS production, we measured ROS levels. Our results showed that CDK5RAP1 deficiency induced cell ROS generation in MCF-7 cells significantly (Fig. 3E-G).

Various apoptotic stimuli can rapidly activate MAPKs, which include p-JNK (15). The activation of JNK is associated with ROS elevation (16). A previous study suggested that activation of JNK through ROS generation is important for apoptosis (33). To investigate this hypothesis, we examined the expression of p-JNK in the CDK5RAP1-deficient MCF-7 cells. The p-JNK activated through ROS-dependent pathway induces the overexpression of tumor suppressors, such as p53 (17). p53, a tumor suppressor protein, triggers cell cycle arrest to provide time for self-mediated apoptosis through transcriptional activation of cyclin-dependent kinase inhibitor (34). In addition to cell cycle arrest, p53 can induce the expression of several factors involved in apoptosis, such as caspase-9 and caspase-3 (35). The activation of caspase-9 and caspase- 3 damage the cell structure and cause functional disorder by proteolysis, final induction of apoptosis (36). Our data demonstrated that the expression of p-JNK, p53, caspase-9 and caspase-3 were all upregulated in CDK5RAP1-deficient MCF-7 cells (Fig. 4). This suggests that p-JNK, p53, caspase-9 and caspase- 3 are all involved in the apoptosis process. As shown in Fig. 5, pretreatment with NAC (the inhibitor of ROS) or SP600125 (the inhibitor of JNK), prevented the apoptosis and the high expression of p-JNK, p53, caspase-9 and caspase-3 in CDK5RAP1-deficient MCF-7 cells. These results clearly indicate that CDK5RAP1 deficiency induces the mitochondrial apoptosis by the ROS/JNK signaling pathway.

CDK5RAP1 deficiency induces cell cycle arrest and apoptosis in MCF-7 cells via ROS generation, resulting p-JNK and p53 activation, increase in cleavage of caspase- 9 and caspase-3, according to the mechanism described in Fig. 6. Although our data provided evidence that tumor growth was markedly inhibited in the CDK5RAP1-deficient MCF-7 cells, the complex process and mechanism require further investigation in the future.

In the present study, to the best of our knowledge, we demonstrated for the first time that CDK5RAP1 deficiency induces cell cycle arrest and apoptosis in human breast cancer MCF-7 cells by the ROS/JNK signaling pathway. The potential of CDK5RAP1 deficiency in cancer cells is expected to provide key insight into the development of novel clinical treatments for cancer.

\section{References}

1. Atta M, Mulliez E, Arragain S, Forouhar F, Hunt JF and Fontecave M: S-Adenosylmethionine-dependent radical-based modification of biological macromolecules. Curr Opin Struct Biol 20: 684-692, 2010.

2. Kaminska KH, Baraniak U, Boniecki M, Nowaczyk K, Czerwoniec A and Bujnicki JM: Structural bioinformatics analysis of enzymes involved in the biosynthesis pathway of the hypermodified nucleoside ms $\left({ }^{2}\right)$ io $\left({ }^{6}\right)$ A37 in tRNA. Proteins 70: $1-18,2008$.

3. Pierrel F, Douki T, Fontecave M and Atta M: MiaB protein is a bifunctional radical-S-adenosylmethionine enzyme involved in thiolation and methylation of tRNA. J Biol Chem 279: 47555-47563, 2004.

4. Bouadloun F, Srichaiyo T, Isaksson LA and Bjork GR: Influence of modification next to the anticodon in tRNA on codon context sensitivity of translational suppression and accuracy. J Bacteriol 166: 1022-1027, 1986.

5. Jenner LB, Demeshkina N, Yusupova G and Yusupov M: Structural aspects of messenger RNA reading frame maintenance by the ribosome. Nat Struct Mol Biol 17: 555-560, 2010.

6. Gong CX and Iqbal K: Hyperphosphorylation of microtubuleassociated protein tau: a promising therapeutic target for Alzheimer disease. Curr Med Chem 15: 2321-2328, 2008.

7. Esberg B, Leung HC, Tsui HC, Bjork GR and Winkler ME: Identification of the miaB gene, involved in methylthiolation of isopentenylated A37 derivatives in the tRNA of Salmonella typhimurium and Escherichia coli. J Bacteriol 181: 7256-7265, 1999.

8. Globisch D, Pearson D, Hienzsch A, Brückl T, Wagner M, Thoma I, Thumbs P, Reiter V, Kneuttinger AC, Müller M, Sieber SA and Carell T: Systems-based analysis of modified tRNA bases. Angew Chem Int Ed Engl 50: 9739-9742, 2011.

9. Brody JG, Rudel RA, Michels KB, Moysich KB, Bernstein L, Attfield KR and Gray S. Environmental pollutants, diet, physical activity, body size, and breast cancer: where do we stand in research to identify opportunities for prevention? Cancer 109: 2627-2634, 2007.

10. Fulda S and Debatin KM: Targeting apoptosis pathways in cancer therapy. Curr Cancer Drug Targets 4: 569-576, 2004.

11. Gamet-Payrastre L, Li P, Lumeau S, Cassar G, Dupont MA, Chevolleau S, Gasc N, Tulliez J and Tercé F: Sulforaphane, a naturally occurring isothiocyanate, induces cell cycle arrest and apoptosis in HT29 human colon cancer cells. Cancer Res 60: 1426-1433, 2000

12. Snoek BC, de Wilt LH, Jansen G and Peters GJ: Role of E3 ubiquitin ligases in lung cancer. World J Clin Oncol 4: 58-69, 2013.

13. Myatt SS, Brosens JJ and Lam EW: Sense and sensitivity: FOXO and ROS in cancer development and treatment. Antioxid Redox Signal 14: 675-687, 2011.

14. Trachootham D, Alexandre J and Huang P: Targeting cancer cells by ROS-mediated mechanisms: a radical therapeutic approach? Nat Rev Drug Discov 8: 579-591, 2009.

15. Kong D, Zheng T, Zhang M, Wang D, Du S, Li X, Fang J and Cao X: Static mechanical stress induces apoptosis in rat endplate chondrocytes through MAPK and mitochondria-dependent caspase activation signaling pathways. PLoS One 8: e69403, 2013.

16. Uchakina ON, Ban $\mathrm{H}$ and McKallip RJ: Targeting hyaluronic acid production for the treatment of leukemia: treatment with 4-methylumbelliferone leads to induction of MAPK-mediated apoptosis in K562 leukemia. Leuk Res 37: 1294-1301, 2013.

17. Leber B, Geng F, Kale J and Andrews DW: Drugs targeting Bcl-2 family members as an emerging strategy in cancer. Expert Rev Mol Med 12: e28, 2010.

18. Yuan Z, Feng W, Hong J, Zheng Q, Shuai J and Ge Y: p38MAPK and ERK promote nitric oxide production in cultured human retinal pigmented epithelial cells induced by high concentration glucose. Nitric Oxide 20: 9-15, 2009.

19. Pozarowski P and Darzynkiewicz Z: Analysis of cell cycle by flow cytometry. Methods Mol Biol 281: 301-311, 2004.

20. Rastogi RP, Singh SP, Häder DP and Sinha RP: Detection of reactive oxygen species (ROS) by the oxidant-sensing probe 2 , 7'-dichlorodihydrofluorescein diacetate in the cyanobacterium Anabaena variabilis PCC 793. Biochem Biophys Res Commun 397: 603-607, 2010.

21. Laemmli UK: Cleavage of structural proteins during the assembly of the head of bacteriophage T4. Nature 227: 680-685, 1970. 
22. Kyhse-Andersen J: Electroblotting of multiple gels: a simple apparatus without buffer tank for rapid transfer of proteins from polyacrylamide to nitrocellulose. J Biochem Biophys Methods 10: 203-209, 1984.

23. Lan T, Wang L, Xu Q, Liu W, Jin H, Mao W, Wang $X$ and Wang X: Growth inhibitory effect of Cucurbitacin E on breast cancer cells. Int J Clin Exp Pathol 6: 1799-1805, 2013.

24. Drexler HG: Review of alterations of the cyclin-dependent kinase inhibitor INK4 family genes p15, p16, p18 and p19 in human leukemia-lymphoma cells. Leukemia 12: 845-859, 1998.

25. Padua MB and Hansen PJ: Changes in expression of cell-cyclerelated genes in PC-3 prostate cancer cells caused by ovine uterine serpin. J Cell Biochem 107: 1182-1188, 2009.

26. Wyllie AH, Kerr JF and Currie AR: Cell death: the significance of apoptosis. Int Rev Cytol 68: 251-306, 1980.

27. Cooper WA, Kohonen-Corish MR, Zhuang L, McCaughan B, Kennedy C, Screaton G, Sutherland RL and Lee CS: Role and prognostic significance of tumor necrosis factor-related apoptosis-inducing ligand death receptor DR5 in nonsmall-cell lung cancer and precursor lesions. Cancer 113: 135-142, 2008.

28. Dewaele M, Maes H and Agostinis P: ROS-mediated mechanisms of autophagy stimulation and their relevance in cancer therapy. Autophagy 6: 838-854, 2010 .

29. Madureira PA, Hill R, Miller VA, Giacomantonio C, Lee PW and Waisman DM: Annexin A2 is a novel cellular redox regulatory protein involved in tumorigenesis. Oncotarget 2: 1075-1093, 2011.
30. Kirshner JR, He S, Balasubramanyam V, Kepros J, Yang CY, Zhang M, Du Z, Barsoum J and Bertin J: Elesclomol induces cancer cell apoptosis through oxidative stress. Mol Cancer Ther 7: 2319-2327, 2008.

31. You BR and Park WH: Zebularine-induced apoptosis in Calu-6 lung cancer cells is influenced by ROS and GSH level changes. Tumour Biol 34: 1145-1153, 2013.

32. Strickertsson JA, Desler C, Martin-Bertelsen T, Machado AM, Wadstrøm T, Winther O, Rasmussen LJ and Friis-Hansen L: Enterococcus faecalis infection causes inflammation, intracellular oxphos-independent ROS production, and DNA damage in human gastric cancer cells. PLoS One 8: e63147, 2013.

33. Lee SJ, Kim MS, Park JY, Woo JS and Kim YK: 15-Deoxy-delta 12,14-prostaglandin J2 induces apoptosis via JNK-mediated mitochondrial pathway in osteoblastic cells. Toxicology 248: 121-129, 2008.

34. Lane DP: Cancer. p53, guardian of the genome. Nature 358: 15-16, 1992.

35. Wang Q, Su L, Liu N, Zhang L, Xu W and Fang H: Cyclin dependent kinase 1 inhibitors: a review of recent progress. Curr Med Chem 18: 2025-2043, 2011.

36. Lee K, Hart MR, Briehl MM, Mazar AP and Tome ME: The copper chelator ATN-224 induces caspase-independent cell death in diffuse large B cell lymphoma. Int J Oncol 45: 439-447, 2014. 